\title{
Electron Detachment in Negative-Ion Collisions. I. Time- dependent theory and Models for a Propagator
}

\author{
T. S. Wang \\ John B. Delos \\ William \& Mary, jbdelos@wm.edu
}

Follow this and additional works at: https://scholarworks.wm.edu/aspubs

Part of the Physics Commons

\section{Recommended Citation}

Wang, T. S. and Delos, John B., Electron Detachment in Negative-lon Collisions. I. Time-dependent theory and Models for a Propagator (1984). Physical Review A, 29(2), 542-551.

https://doi.org/10.1103/PhysRevA.29.542

This Article is brought to you for free and open access by the Arts and Sciences at W\&M ScholarWorks. It has been accepted for inclusion in Arts \& Sciences Articles by an authorized administrator of W\&M ScholarWorks. For more information, please contact scholarworks@wm.edu. 


\title{
Electron detachment in negative-ion collisions. I. Time-dependent theory and models for a propagator
}

\author{
T. S. Wang and J. B. Delos \\ Physics Department, The College of William and Mary, Williamsburg, Virginia 23185
}

(Received 23 June 1983)

\begin{abstract}
A theory of electron detachment in slow collisions of negative ions with atoms is developed. The theory is based on assumptions that are similar to, but more general than, the assumptions made in earlier papers: The motion of the nuclei is described semiclassically, the electronic wave function is expanded in a partially diabatic basis that includes a discrete state and a continuum, and certain couplings are assumed to be small. With such assumptions the Schrödinger equation is reduced to a nondenumerably infinite set of coupled differential equations, and then to a single integrodifferential equation (an equation with "memory"). It is shown that the solution depends upon two functions, the energy gap $\Delta(t)$ between the discrete state and the continuum, and a propagator $\mathscr{G}\left(t, t^{\prime}\right)$. General properties of the propagator are given and it is calculated for a very simple model. Formal properties of the integro-differential equation are also investigated.
\end{abstract}

\section{INTRODUCTION}

This is one of a series of papers ${ }^{1}$ dealing with electron detachment in collisions of negative ions with atoms,

$$
A^{-}+B \rightarrow A+B+e^{-} \text {. }
$$

The theory developed here can also be used to study various types of ionization processes, such as Penning ionization,

$$
A^{*}+B \rightarrow A+B^{+}+e^{-}
$$

and dissociative recombination and dissociative attachment,

$$
\begin{aligned}
& e^{-}+A B^{+} \rightarrow A+B, \\
& e^{-}+A B \rightarrow A^{-}+B,
\end{aligned}
$$

but in these papers we focus on the first process, for which there is a wealth of recent experimental data. ${ }^{2-5}$

In an earlier paper ${ }^{1(a)}$ the theory was developed starting from a semiclassical close-coupling framework, following ideas of Fano ${ }^{6}$ and Demkov. ${ }^{7}$ Certain assumptions were made about matrix elements, and then the close-coupled equations were solved by taking a Fourier transform from time into energy. In the present paper we start from similar basic assumptions, but we develop the mathematical aspects in a different way; the result is a formulation that is simpler, more general, and in some ways more transparent than the earlier formulation.

More specifically, it has been shown that the results of the collision depend mainly upon the energy gap between ion and neutral curves as a function of time, $\Delta(t)$, and upon the coupling between bound and free states, $V_{-1, \epsilon}(t)$. In Ref. 1(a) we neglected the time dependence of the coupling $V_{-1 \epsilon}$, and we took $\Delta(t)$ to be a quadratic function of time. Obviously one wants a formulation that does not lean too heavily on such specific approximations. Howev- er, when one tries to generalize the theory given in Ref. 1(a), one finds that the (Fourier transformed) equations become quite complicated, and it is not clear how to obtain a solution to them. In the present paper we show that the desired generalizations can be achieved by retaining the time $t$ as the independent variable, and working with an integro-differential equation.

\section{FORMULATION OF THE THEORY}

In this section we present the assumptions on which the theory is based, and we develop the equations which are to be solved.

\section{A. Hypotheses}

We begin by describing the nuclear motion semiclassically, so that the wave function for the electrons satisfies a time-dependent Schrödinger equation,

$$
h(\overrightarrow{\mathrm{r}} ; \overrightarrow{\mathrm{R}}(t)) \Upsilon(\overrightarrow{\mathrm{r}}, t)=i \hbar \frac{\partial \Upsilon(\overrightarrow{\mathrm{r}}, t)}{\partial t} .
$$

If the electronic wave function is expanded in an orthonormal set of basis functions

$$
\Upsilon(\overrightarrow{\mathrm{r}}, t)=\underset{n}{f_{n}} b_{n}(t) \phi_{n}(\overrightarrow{\mathrm{r}} ; \overrightarrow{\mathrm{R}}(t)),
$$

where $n$ can be a discrete or continuous index, then the vector of coefficients $\underline{b}(t)$ satisfies the coupled equations

$$
i \hbar \frac{d}{d t} \underline{b}(t)=\left(\underline{\mathscr{C}}+v^{R} \underline{P}^{R}\right) \underline{b}(t)
$$

where

$$
\begin{aligned}
& \underline{\mathscr{V}}=\underline{h}+v^{\theta} \underline{P}^{\theta}+v^{\Phi} \underline{P}^{\Phi}, \\
& h_{m n}=\left\langle\phi_{m}(\overrightarrow{\mathrm{r}} ; \overrightarrow{\mathrm{R}})|h| \phi_{n}(\overrightarrow{\mathrm{r}} ; \overrightarrow{\mathrm{R}})\right\rangle, \\
& \overrightarrow{\mathbf{P}}_{m n}=\left\langle\phi_{m}(\overrightarrow{\mathrm{r}} ; \overrightarrow{\mathrm{R}})\left|-i \hbar \vec{\nabla}_{R}\right| \phi_{n}(\overrightarrow{\mathrm{r}} ; \overrightarrow{\mathrm{R}})\right\rangle .
\end{aligned}
$$


In principle, the expansion (2.2) should include electron translation factors ${ }^{8}$ (ETF's). However, since we are mainly interested in such systems as $\mathrm{H}^{-}-\mathrm{He}$, in which the active electron is clearly associated with and propagating with a single center, the most important effects of ETF's can be incorporated by taking the relevant nucleus to be the center for the electron coordinates. A more sophisticated approach would be needed for symmetric systems (such as $\mathrm{H}^{-}-\mathrm{H}$ ) or for any system in which real or virtual charge exchange is important, but even in such cases, the effects of ETF's in slow collisions are probably not very great. ${ }^{9}$

Let the basis set consist of one discrete state $\phi_{-1}(\vec{r} ; \vec{R})$, which describes the electron bound to $A B$, and a continuum of free states, $\phi_{\epsilon}(\vec{r} ; \vec{R})$, representing the electron escaping from $A B$ with asymptotic kinetic energy $\epsilon$, which typically will be a few electron volts or less. Starting from any such orthonormal basis, it is possible to make transformations to representations which are partially diabatic and partially adiabatic. Let us select a representation in which the couplings between the bound state and the significant (low energy) part of the continuum are represented diabatically, i.e., such that the radial nonadiabatic coupling matrix element is negligible,

$$
v^{R} P_{-1, \epsilon}^{R}(R) \simeq 0
$$

and couplings between the discrete state and the continuum are represented by matrix elements $\mathscr{V}_{-1, \epsilon}(R)$. That such a representation exists is shown in Appendix A. The present theory is based upon models for the behavior of the matrix elements in this representation.

An important simplification in the present theory is the assumption that the coupling within the continuum itself is negligible,

$$
\mathscr{V}_{\epsilon \epsilon^{\prime}} \simeq 0, \quad v^{R} P_{\epsilon \epsilon^{\prime}}^{R} \simeq 0, \quad \epsilon \neq \epsilon^{\prime}
$$

so transitions occur from the bound state to the free states, and vice versa, but direct transitions from one free state to another are neglected. Since this approximation was not adequately explained in Ref. 1, we give some discussion of it here.

When a low-energy electron collides with a molecule, it is well known that vibrational excitation is very unlikely unless the electron is temporarily captured. In classical language, the light electron cannot have much impact upon the heavy nuclei, and it cannot easily cause them to change their state of motion unless it is allowed to interact with them for a long time. Conversely, in any collision that does not involve temporary capture of the electron, slow motion of the heavy particles does not significantly affect the electronic state, and the electron scatters from the molecule almost as if the nuclei were fixed.

In quantum mechanics, this idea is expressed in the "adiabatic nuclei" approximation, ${ }^{10}$ in which the full wave function for electrons and nuclei is taken to be a product

$$
\psi(\overrightarrow{\mathbf{R}}, \overrightarrow{\mathrm{r}}) \simeq \phi_{\overrightarrow{\mathrm{k}}}(\overrightarrow{\mathrm{r}} ; \overrightarrow{\mathbf{R}}) \chi_{V J M}(\overrightarrow{\mathbf{R}}) .
$$

Taken by itself, such a product form implies that the nuclei have a definite (fixed) vibrational and rotational state
$(V, J, M)$ and that the electrons sit in a state that adjusts continuously to the nuclear position. Vibrational and rotational transitions are not contained in this simpleproduct wave function, but they can be calculated from it by taking matrix elements of the form

$$
\int d \overrightarrow{\mathrm{r}} d \overrightarrow{\mathbf{R}} e^{-i \overrightarrow{\mathrm{k}} \cdot \overrightarrow{\mathrm{r}}} \chi_{V^{\prime} J^{\prime} M^{\prime}}^{*}(\overrightarrow{\mathbf{R}}) U(\overrightarrow{\mathrm{r}}, \overrightarrow{\mathbf{R}}) \phi_{\overrightarrow{\mathrm{k}}}(\overrightarrow{\mathrm{r}} ; \overrightarrow{\mathbf{R}}) \chi_{V J M}(\overrightarrow{\mathbf{R}}),
$$

where $U(\vec{r}, \vec{R})$ is the potential energy of interaction between the incident electron and the molecule. In applying this approximation, normally $\left|\overrightarrow{\mathbf{k}}^{\prime}\right|$ is set equal to $|\overrightarrow{\mathbf{k}}|$; hence although the vibrational and rotational energy changes somewhat, the corresponding change in the electronic state is neglected.

The adiabatic-nuclei approximation is thought to be adequate for cases in which there is no temporary capture of the electron. ${ }^{10}$ For cases in which the electron is captured (as, for example, in $e^{-}-\mathrm{N}_{2}$ scattering near $2 \mathrm{eV}$ ), the adiabatic-nuclei approximation has been used ${ }^{11}$ to describe the "background" scattering, i.e., that part of the scattering that is not related to capture.

In the present paper, we are concerned with collisional detachment of electrons, in which there is an exchange of heavy-particle translational energy with electronic energy. We have distinguished between a discrete state, representing an electron captured by the molecule $A B$, and a continuum, representing scattering other than by capture. According to the above argument, the nuclear motion probably does not have a large effect upon electrons in the continuum states: An escaping electron would move away from the molecule as if the nuclei were fixed, and the slow motion of the nuclei would not significantly change the electron's direction or energy. In our language, then, direct free-to-free transitions should be much less significant than free-to-bound transitions, and it should be reasonable to neglect them. ${ }^{12,13}$

Under these assumptions, the coupled equations (2.3) take a simple form. Defining

$$
\begin{aligned}
& C_{-1}(t)=b_{-1}(t) \exp \left[\frac{i}{\hbar} \int_{t_{0}}^{t} \mathscr{V}_{\text {neutral }}\left(t^{\prime}\right) d t^{\prime}\right], \\
& C_{\epsilon}(t)=b_{\epsilon}(t) \exp \left[\frac{i}{\hbar} \int_{t_{0}}^{t} \mathscr{V}_{\text {neutral }}\left(t^{\prime}\right) d t^{\prime}\right],
\end{aligned}
$$

where $\mathscr{V}_{\text {neutral }}(t)$ is the energy of the lowest state in the continuum, we have

$$
\begin{aligned}
& i \hbar \frac{d C_{-1}(t)}{d t}=\Delta(t) C_{-1}(t)+\int_{0}^{\infty} V_{-1, \epsilon}(t) C_{\epsilon}(t) \rho_{\epsilon} d \epsilon \\
& i \hbar \frac{d C_{\epsilon}(t)}{d t}=V_{\epsilon \epsilon}(t) C_{\epsilon}(t)+V_{\epsilon,-1}(t) C_{-1}(t)
\end{aligned}
$$

where

$$
\begin{aligned}
& \Delta(t)=\mathscr{V}_{-1,-1}(t)-\mathscr{V}_{\text {neutral }}(t), \\
& V_{\epsilon \epsilon}(t)=\mathscr{V}_{\epsilon \epsilon}(t)-\mathscr{V}_{\text {neutral }}(t), \\
& V_{\epsilon,-1}(t)=\mathscr{V}_{\epsilon,-1}(t) .
\end{aligned}
$$

Equation (2.8) is a nondenumerably infinite set of coupled 
equations, which are the starting point of the present theory. It should be emphasized that these equations are not new: They were earlier used by Demkov and his collaborators, ${ }^{7}$ and the quantum version of them was briefly discussed by Fiquet-Fayard. ${ }^{14}$ Our contribution is a new method for solving these equations, and the application of this method to certain collision systems.

Up to this point, the assumptions that have been made are the following: (i) semiclassical treatment of nuclear motion; (ii) one discrete state and one continuum; (iii) orthogonal diabatic representation; and (iv) neglect of intracontinuum couplings. These assumptions are regarded as defining "generic" properties of a broad class of systems. Specific forms of the matrix elements-i.e., the time dependence and $\epsilon$ dependence of $\Delta(t), V_{\epsilon \epsilon}(t)$, and $V_{\epsilon,-1}(t)$-are regarded as "constitutive" properties, which depend upon the particular system being considered. Of course it is assumed that all of the matrix elements are bounded, continuous, and differentiable functions of $\epsilon$ and $t$; also, a sufficient condition for validity of our manipulations is that

$$
\int_{0}^{\infty} d \epsilon \int_{t_{0}}^{t} d t^{\prime}\left|V_{-1, \epsilon}\left(t^{\prime}\right) V_{\epsilon,-1}\left(t^{\prime}\right)\right| \rho_{\epsilon}\left(t^{\prime}\right)
$$

converges for all finite $t_{0}, t$, and in the limits $t_{0} \rightarrow-\infty, t \rightarrow+\infty$.

In the work of Taylor and Delos ${ }^{1(a)}$ several additional assumptions were made. (v) Diagonal continuum matrix elements form parallel curves, so $V_{\epsilon \epsilon}(R)-V_{\epsilon^{\prime} \epsilon^{\prime}}(R)$ has a negligible dependence on $R$, and

$$
\mathscr{V}_{\epsilon \epsilon}(R)=V_{\text {neutral }}(R)+\epsilon, \quad 0<\epsilon<\infty
$$

with $\epsilon$ independent of $R$. Also the density of states $\rho_{\epsilon}$ is independent of $R$. (vi) The $R$ dependence (or $t$ dependence) of the coupling matrix element $V_{\epsilon,-1}$ between the bound and free states can be neglected. (vii) The energy gap $\Delta(t)$ is approximately a quadratic function of time.

Later, in our calculations, we will again use assumption (v); nothing in our general formulation really depends upon this assumption, but we will show that it is almost always possible to define basis states in the continuum such that their energy curves are parallel. Later also, instead of (vi), we will use the approximation that all bound-free coupling matrix elements have the same time dependence

$$
V_{-1, \epsilon}(t) \approx \hat{V}_{-1, \epsilon} g(t) .
$$

This approximation should be reasonably accurate for most systems undergoing electron detachment, and it is very convenient; it simplifies the formulas and the calculations, but we emphasize that it is not essential to the present theory. Assumption (vii) is abandoned, and we allow in principle a general time dependence of $\Delta(t)$. For example, in some calculations ${ }^{1(\mathrm{e})}$ (to be reported later) we have used a quartic approximation to $\Delta(t)$.

\section{B. Uncoupling the equations}

The infinite set of coupled equations (2.8) is easily reduced to a single equation because it is possible to solve Eq. (2.8b) for $C_{\epsilon}(t)$ in terms of $C_{-1}(t)$

$$
C_{\epsilon}(t)=\exp \left[-i \Phi_{\epsilon}\left(t, t_{0}\right) / \hbar\right]\left[C_{\epsilon}\left(t_{0}\right)+(i \hbar)^{-1} \int_{t_{0}}^{t} d t^{\prime} V_{\epsilon,-1}\left(t^{\prime}\right) \exp \left[i \Phi_{\epsilon}\left(t^{\prime}, t_{0}\right) / \hbar\right] C_{-1}\left(t^{\prime}\right)\right],
$$

where

$$
\Phi_{\epsilon}\left(t_{2}, t_{1}\right)=\int_{t_{1}}^{t_{2}} V_{\epsilon \epsilon}(t) d t
$$

Substituting (2.13) into [2.8(a)] we obtain

$$
\begin{aligned}
i \hbar \frac{d C_{-1}(t)}{d t}= & \Delta(t) C_{-1}(t)+\left[(i \hbar)^{-1} \int_{0}^{\infty} d \epsilon \rho_{\epsilon} V_{-1, \epsilon}(t) \exp \left[-i \Phi_{\epsilon}\left(t, t_{0}\right) / \hbar\right] \int_{t_{0}}^{t} d t^{\prime} V_{\epsilon,-1}\left(t^{\prime}\right) \exp \left[i \Phi_{\epsilon}\left(t^{\prime}, t_{0}\right) / \hbar\right] C_{-1}\left(t^{\prime}\right)\right] \\
& +\int_{0}^{\infty} d \epsilon \rho_{\epsilon} V_{-1, \epsilon}(t) \exp \left[-i \Phi_{\epsilon}\left(t, t_{0}\right) / \hbar\right] C_{\epsilon}\left(t_{0}\right),
\end{aligned}
$$

and, reversing the order of integration [valid if $(2.10)$ converges]

$$
\left\lceil i \hbar \frac{d}{d t}-\Delta(t)\right] C_{-1}(t)-\int_{t_{0}}^{t} \mathscr{G}\left(t, t^{\prime}\right) C_{-1}\left(t^{\prime}\right) d t^{\prime}=\mathscr{I}(t),
$$

where

$$
\begin{aligned}
\mathscr{G}\left(t, t^{\prime}\right)=(i \hbar)^{-1} \int_{0}^{\infty} & d \epsilon \rho_{\epsilon} V_{-1, \epsilon}(t) \\
& \times \exp \left[-i \Phi_{\epsilon}\left(t, t^{\prime}\right) / \hbar\right] V_{\epsilon,-1}\left(t^{\prime}\right),
\end{aligned}
$$

$$
\mathscr{I}(t)=\int_{0}^{\infty} d \epsilon \rho_{\epsilon} V_{-1, \epsilon}(t) \exp \left[-i \Phi_{\epsilon}\left(t, t_{0}\right) / \hbar\right] C_{\epsilon}\left(t_{0}\right)
$$

\section{Boundary conditions and formal properties}

There are two sets of boundary conditions that apply to normal collisions. If the system begins in the bound state,

$$
C_{-1}\left(t_{0}\right)=1, C_{\epsilon}\left(t_{0}\right)=0,
$$

from which it follows that

$$
\mathscr{I}(t)=0 \text {. }
$$


If the system begins in one of the free states,

$$
C_{-1}\left(t_{0}\right)=0, \quad C_{\epsilon}\left(t_{0}\right)=\delta\left(\epsilon-\epsilon_{0}\right) / \rho_{\epsilon_{0}},
$$

then

$$
\mathscr{I}(t)=V_{-1, \epsilon_{0}}(t) \exp \left[-i \Phi_{\epsilon_{0}}\left(t, t_{0}\right) / \hbar\right] .
$$

In the present paper, we will only consider the boundary conditions (2.19), so $C_{-1}(t)$ satisfies the homogeneous counterpart to (2.16),

$$
\left[i \hbar \frac{d}{d t}-\Delta(t)\right] C_{-1}(t)-\int_{t_{0}}^{t} \mathscr{G}\left(t, t^{\prime}\right) C_{-1}\left(t^{\prime}\right) d t^{\prime}=0 .
$$

The matrices $\underline{h}, \underline{P}, \underline{\mathscr{V}}$, and $\underline{V}$ are all Hermitian, so the total probability for finding the system in bound and free states is conserved,

$$
\left|C_{-1}(t)\right|^{2}+\int_{0}^{\infty}\left|C_{\epsilon}(t)\right|^{2} \rho_{\epsilon} d \epsilon=1
$$

The quantities $\mathscr{G}\left(t, t^{\prime}\right), \quad \partial \mathscr{G}\left(t, t^{\prime}\right) / \partial t, \mathscr{I}(t), d \mathscr{I} / d t$, $C_{-1}(t), d C_{-1}(t) / d t$, and $d^{2} C_{-1}(t) / d t^{2}$ all are continuous functions of $t$, provided that certain integrals converge. Proofs are given in Appendix B. These seemingly dull mathematical properties turn out to be very important and fundamental; later we will show, for example, that because the second derivative is continuous, the local-complexpotential model cannot be exactly correct, for it would give a discontinuous second derivative to $C_{-1}(t)$.

In the limit as $t \rightarrow \infty, C_{-1}(t)$ is oscillatory, but $\left|C_{-1}(t)\right|$ and $\left|C_{\epsilon}(t)\right|$ must approach definite limits; after all, $\left|C_{-1}(\infty)\right|^{2}$ is supposed to be the survival probability for the negative ion, and $\left|C_{\epsilon}(\infty)\right|^{2} \rho_{\epsilon}$ is the probability that an electron will be detached with energy near $\epsilon$. We show in Appendix $\mathrm{C}$ that if $\int_{t_{0}}^{t} \mathscr{G}\left(t, t^{\prime}\right) d t^{\prime}$ goes to zero sufficiently rapidly as $t \rightarrow \infty$, then

$$
\widehat{C}_{-1}(t) \equiv C_{-1}(t) \exp \left[-i \int_{t_{0}}^{t} \Delta\left(t^{\prime}\right) d t^{\prime} / \hbar\right]
$$

approaches a finite limit as $t \rightarrow \infty$.

\section{Discussion}

The complete solution to the coupled equations (2.8) under the boundary condition (2.19) is now accomplished in three steps. (1) Evaluate the propagator $\mathscr{G}\left(t, t^{\prime}\right)$ defined in Eq. (2.17); (2) solve the fundamental integro-differential equation (2.23) for $C_{-1}(t)$; (3) use $C_{-1}(t)$ in Eq. (2.13) to obtain $C_{\epsilon}(t) .{ }^{15}$ In the next section we derive the general properties of the propagator $\mathscr{G}\left(t, t^{\prime}\right)$, and we calculate its value for a specific (and rather oversimplified) model.

\section{THE PROPAGATOR}

\section{A. Introduction}

According to Eq. (2.23), collisions in which the electron is originally bound are governed by two functions, $\Delta(t)$ and $\mathscr{G}\left(t, t^{\prime}\right)$. In principle, both of these functions can be obtained from $a b$ initio calculations. $\Delta(t)$ is the gap between the negative-ion and neutral-molecule energy curves, and this has been calculated for a number of systems. ${ }^{16}$ However, a more elaborate calculation would be required to obtain $\mathscr{G}\left(t, t^{\prime}\right)$.

The properties of $\mathscr{G}\left(t, t^{\prime}\right)$ follow from those of the phase function $\Phi_{\epsilon}\left(t, t^{\prime}\right)$, the density of states $\rho_{\epsilon}(t)$, and the coupling matrix element $V_{-1, \epsilon}(t)$. In the next section we shall examine each of these elements of $\mathscr{G}\left(t, t^{\prime}\right)$. Then in Sec. III C, we give general properties of $\mathscr{G}\left(t, t^{\prime}\right)$ and we present an important simplifying approximation, which we call a "short-memory" approximation. In Sec. III D a reduced propagator $\hat{Y}(\tau)$ is given under a "separable" approximation, and in Sec. III E we show some model calculations of $\hat{\mathscr{G}}(\tau)$ and its Fourier transform.

For the benefit of readers who do not want to know the details, let us state the main result: Under conditions discussed in this section, $\mathscr{G}\left(t, t^{\prime}\right)$ can be written approximately as

$$
\mathscr{G}\left(t, t^{\prime}\right) \simeq|g(t)|^{2} \hat{\mathscr{G}}\left(t-t^{\prime}\right),
$$

where $\hat{\mathscr{G}}\left(t-t^{\prime}\right) \equiv \hat{\mathscr{Y}}(\tau)$ has a shape like that shown in Fig. 1, and $g(t)$ has a shape like that shown in Fig. 2. Also the Fourier transform of $\hat{G}(\tau)$ is called $\hat{G}(\epsilon)$, and it has a shape like those shown in Figs. 3 and 4. The real and imaginary parts of $\hat{G}(\epsilon)$ are, respectively, related to the "level shift" and "width." This is all we need to know in order to understand the calculations given in the following paper.

\section{B. Elements in $\mathscr{G}\left(t, t^{\prime}\right)$}

\section{Phase function}

If assumption (v) of Sec. III A holds, i.e., if the basis functions $\phi_{\epsilon}(\overrightarrow{\mathbf{r}} ; \overrightarrow{\mathbf{R}})$ are chosen such that their energies $\mathscr{V}_{\epsilon \epsilon}(R)$ form a set of parallel curves, then we can take $\epsilon$ to be that energy relative to the lowest state in the continuum

$$
\epsilon=\mathscr{V}_{\epsilon \epsilon}(R)-\mathscr{V}_{\text {neutral }}(R), \quad 0<\epsilon<\infty
$$

and $\epsilon$ will be independent of $R$. Hence from Eqs. (2.14) and $(2.9 \mathrm{~b})$ we have

$$
\Phi_{\epsilon}\left(t, t^{\prime}\right)=\left(t-t^{\prime}\right) \epsilon .
$$

It is difficult to imagine a representation for a negative-ion system in which Eq. (3.3) would not hold. To understand this, suppose (to simplify the argument) that the detached electron occupies $s$ waves only. Then

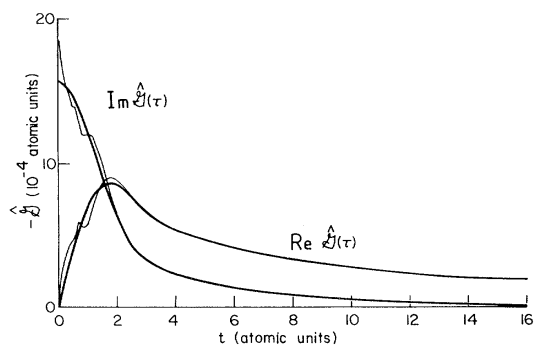

FIG. 1. Reduced propagator $\mathscr{G}(\tau)$ for the square-well model defined in Ref. 1(a). 


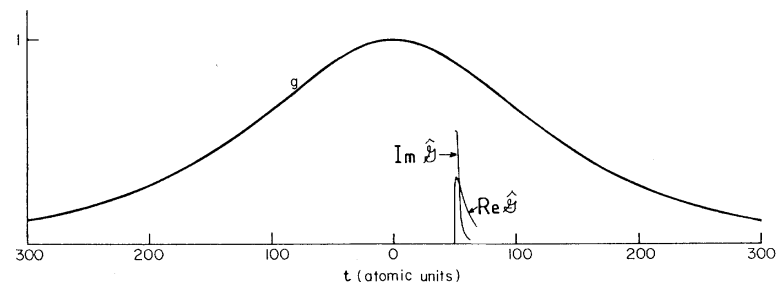

FIG. 2. Typical behavior of the dimensionless quantity $g(t)$, which multiplies the reduced propagator [Eqs. (3.1) and (3.21)]. (This graph shows $\exp \left[-\left(b^{2}+v^{2} t^{2}\right)^{1 / 2}\right], b=1, v=0.01$, with $b$ and $v$ representing the impact parameter and internuclear velocity, respectively.) On the same time scale, but using a different scale for the ordinate, is $\hat{\mathscr{G}}\left(t-t^{\prime}\right)$, which is shown in more detail in Fig. 1.

each basis state is a function only of scalar $r$ (not vector $\vec{r})$. Basis functions are normally chosen to be eigenfunctions of some simplified Hamiltonian, and at large distances they would have the asymptotic form

$$
\phi_{n}(r ; R)=\exp (-i k r)-\exp \{i[k r+2 \delta(k ; R)]\},
$$

where $\delta(k ; R)$ is the phase shift, which is a finite, continuous function of $k$ and $R$. The boundary condition $\phi_{N}(r ; R)=0$ at $r=L$ leads to

$$
\begin{array}{ll} 
& k_{N} L+\delta\left(k_{N} ; R\right)=N \pi \\
\text { or } \quad & k_{N}=\left[N \pi-\delta\left(k_{N} ; R\right)\right] / L .
\end{array}
$$

We take the limit $N \rightarrow \infty, L \rightarrow \infty, N / L$ finite. In that limit $k_{N}$ and $\epsilon_{N}=\hbar^{2} k_{N}^{2} / 2 m$ become independent of $R$.

So long as the continuum states have the asymptotic form (3.4), with finite, continuous phase shifts, and provided that boundary conditions are applied in a large box, it follows that the energies of the continuum states will form a set of parallel curves, and that the density of states will be the same as that for a free particle,

$$
\rho_{\epsilon}=(2 m)^{1 / 2} \epsilon^{-1 / 2} L /(2 \pi \hbar),
$$

and of course it is independent of $R$.

With more effort, it is possible to establish the same result in three dimensions, in which case the density of states is

$$
\rho_{k} d k=\frac{m L^{3}}{8 \pi^{3} \hbar^{2}} k \sin \theta d k d \theta d \phi
$$

assuming that the wave functions are normalized to unity

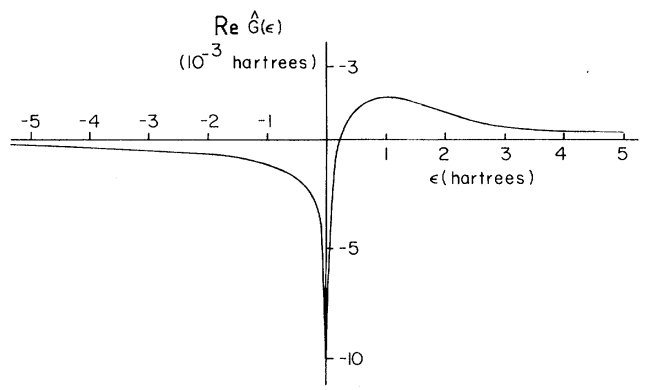

FIG. 3. Real part of the Green function $\widehat{G}(\epsilon)$ for the squarewell model [Ref. 1(a)]. This is the Fourier transform of $\mathscr{Y}(\tau)$, and it is related to the "level shift."

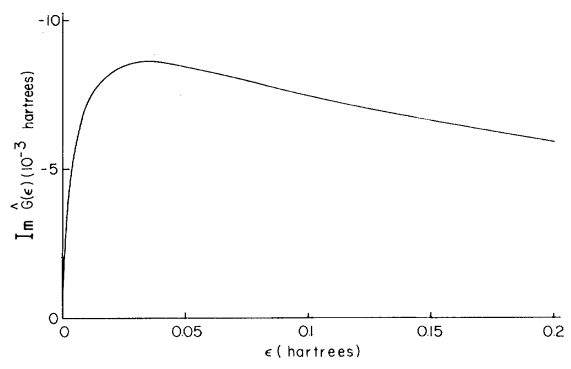

FIG. 4. Imaginary part of $\hat{G}(\epsilon)$ for the square-well model. It is related to the "level width."

in a box of volume $L^{3}$. It should be noted that these results need not apply if long-range (e.g., Coulomb) forces act on the escaping electron, since in that case the phase shift may be infinite.

\section{Coupling matrix element}

The coupling matrix element $V_{-1, \epsilon}(t)$ depends upon the system, the type of coupling (electrostatic, rotational, etc.), and the representation that is chosen. As $t \rightarrow \infty, V_{-1, \epsilon}(t)$ goes to zero faster than $1 / t$, but not faster than $e^{-t / t}$ where $\hat{t}$ is some constant. In principle, $V_{-1, \epsilon}(t)$ can be obtained from an $a b$ initio calculation, using formulas (2.4) and (2.5). There are now some reasonably accurate calculations of wave functions for negative ions, $\phi_{-1}(\vec{r} ; \vec{R})$, but at the present time, practically nothing is known about free states $\phi_{\epsilon}(\vec{r} ; \vec{R})$ representing an electron scattered from an unstable neutral molecule such as HHe. For the time being, we will get the information we need by examining simple models, and by comparing theoretical calculations with experiments.

\section{General properties of $\mathscr{G}\left(t, t^{\prime}\right)$ and short-memory approximation}

Based on the above properties, we can write

$$
\begin{gathered}
\mathscr{G}\left(t, t^{\prime}\right)=(i \hbar)^{-1} \int_{0}^{\infty} V_{-1, \epsilon}(t) \exp \left[-i \epsilon\left(t-t^{\prime}\right) / \hbar\right] \\
\times V_{\epsilon,-1}\left(t^{\prime}\right) \rho_{\epsilon} d \epsilon
\end{gathered}
$$

from which follows the symmetry

$$
\mathscr{G}\left(t^{\prime}, t\right)=-\mathscr{G}^{*}\left(t, t^{\prime}\right) \text {. }
$$

Also, for any fixed $t$

$$
\lim _{t^{\prime} \rightarrow \pm \infty} \mathscr{G}\left(t, t^{\prime}\right)=0
$$

and for any fixed $t^{\prime}$

$$
\lim _{t \rightarrow \pm \infty} \mathscr{G}\left(t, t^{\prime}\right)=0 \text {. }
$$

Since the range of the coupling matrix element $V_{-1, \epsilon}(R)$ is normally a few Bohr radii, it follows that the time scale on which $\mathscr{G}\left(t, t^{\prime}\right)$ is significant is no more than a few atomic length units divided by the collision velocity. For example, consider a slow collision, with the nuclear velocity $\simeq 0.01$ atomic units. In this case $\mathscr{G}\left(t, t^{\prime}\right)$ is negligible 
unless both $|t|$ and $\left|t^{\prime}\right|$ are less than a few hundred atomic time units.

Equation (3.9) also contains $\left(t-t^{\prime}\right)$ in the exponent. To see its effects, let us momentarily ignore the time dependence of $V_{-1, \epsilon}(t)$. Then as $\left|t-t^{\prime}\right| \rightarrow \infty, \mathscr{G}\left(t, t^{\prime}\right) \rightarrow 0$ again. However, this happens on a much shorter time scale: with $\tau=t-t^{\prime}$, the span of time intervals $\Delta \tau$ over which $\mathscr{G}\left(t, t^{\prime}\right)$ is significant is inversely related to the range of energy $\Delta \epsilon$ in which $V_{-1, \epsilon}$ is significant

$$
\Delta \tau \Delta \epsilon \simeq \hbar \text {. }
$$

Since the order of magnitude of $\Delta \epsilon$ would be 1 a.u. of energy $(27.2 \mathrm{eV})$, the order of magnitude of $\Delta \tau$ would be 1 a.u. of time $\left(2.42 \times 10^{-17} \mathrm{sec}\right)$. Thus $\mathscr{G}\left(t, t^{\prime}\right)$ would be negligible for any $t, t^{\prime}$ such that $\left|t-t^{\prime}\right|$ is greater than a few atomic time units.

This important fact provides a useful simplifying approximation for $\mathscr{G}\left(t, t^{\prime}\right)$. The fundamental integrodifferential equation (2.23) may be said to be a differential equation with memory: The time derivative of $C_{-1}(t)$ depends not only upon the present value of $C_{-1}(t)$, but also upon its value at all times in the past. The argument above indicates that the equation's memory is short.

To make use of this, let us rewrite Eq. (3.9) in terms of variables $t$ and $\tau=t-t^{\prime}$ :

$$
\begin{aligned}
\mathscr{G}\left(t, t^{\prime}\right) \equiv \mathscr{G}(\tau ; t) & \\
=(i \hbar)^{-1} \int_{0}^{\infty} & V_{-1, \epsilon}(t) \exp (-i \epsilon \tau / \hbar) \\
& \times V_{\epsilon,-1}(t-\tau) \rho_{\epsilon} d \epsilon .
\end{aligned}
$$

Equation (3.13) is exact. Suppose now $\Delta \tau$ is small compared to the time over which $V_{\epsilon,-1}(t)$ changes significant1y:

$$
\frac{d V_{\epsilon,-1}}{d t} \frac{\Delta \tau}{V_{\epsilon,-1}} \ll 1,
$$

or, using $V_{\epsilon,-1}^{-1} d V_{\epsilon,-1} / d t \simeq v / a_{0}$ and Eq. (3.12),

$$
v \Delta \tau / a_{0} \simeq \hbar v /\left(a_{0} \Delta \epsilon\right)<1 .
$$

If this condition is satisfied, we may substitute $V_{\epsilon,-1}(t-\tau) \simeq V_{\epsilon,-1}(t)$ in Eq. (3.13b) to obtain

$$
\begin{aligned}
\mathscr{G}(\tau ; t) & \simeq \mathscr{G}_{s}(\tau ; t) \\
& =\int_{0}^{\infty}\left|V_{-1, \epsilon}(t)\right|^{2} \exp (-i \epsilon \tau / \hbar) \rho_{\epsilon} d \epsilon / i \hbar
\end{aligned}
$$

$\mathscr{G}_{s}(\tau ; t)$ will be referred to as the short-memory approximation to $\mathscr{G}(\tau ; t)$.

The Fourier transform of $\mathscr{G}(\tau ; t)$ turns out to be very important; it is defined as

$$
G(\epsilon ; t) \equiv \int_{0}^{\infty} \exp (i \epsilon \tau / \hbar) \mathscr{G}(\tau ; t) d \tau .
$$

Using the short-memory approximation, we have

$$
\begin{aligned}
G(\epsilon ; t) & \simeq G_{s}(\epsilon ; t) \\
& \equiv \int_{0}^{\infty} \exp (i \epsilon \tau / \hbar) \mathscr{G}_{s}(\tau ; t) d \tau .
\end{aligned}
$$

If $\epsilon$ is given a small positive imaginary part, using Eq. (3.16), we may reverse the order of integrations over $\tau$ and $\epsilon$ to obtain

$$
G_{s}(\epsilon ; t)=\int_{0}^{\infty}\left|V_{-1, \epsilon}(t)\right|^{2}\left(\epsilon-\epsilon^{\prime}\right)^{-1} \rho_{\epsilon} d \epsilon^{\prime} .
$$

This will appear again in the following paper. For future reference let us also note that for $\epsilon$ close to the positive real axis,

$$
\operatorname{Im} G_{s}(\epsilon, t)=-\pi\left|V_{-1, \epsilon}(t)\right|^{2} \rho_{\epsilon} .
$$

\section{A separable approximation and a reduced propagator}

A further approximation provides physical insight and an additional simplification. Even if the coupling matrix elements are significant over a broad range of the continuum, transitions might only occur into a rather narrow range. For example, if the collision is slow, usually only very-low-energy electrons are produced. Within that narrow range of continuum energies, it may be reasonable to assume that all of the coupling matrix elements have the same time dependence

$$
V_{-1, \epsilon}(t)=g(t) \hat{V}_{-1, \epsilon},
$$

where $\hat{V}_{-1, \epsilon}$ is independent of $t$ and $g(t)$ has a shape as shown in Fig. 2. This gives

$$
\mathscr{G}\left(t, t^{\prime}\right) \simeq \mathscr{G}^{\operatorname{sep}}\left(t, t^{\prime}\right)=g(t) g\left(t^{\prime}\right) \hat{\mathscr{G}}\left(t-t^{\prime}\right),
$$

where

$$
\begin{aligned}
\hat{\mathscr{G}}(\tau) & =\hat{\mathscr{G}}\left(t-t^{\prime}\right) \\
& =(i \hbar)^{-1} \int_{0}^{\infty} d \epsilon \rho_{\epsilon}\left|\hat{V}_{-1, \epsilon}\right|^{2} \exp (-i \epsilon \tau / \hbar)
\end{aligned}
$$

is a reduced propagator. The corresponding shortmemory approximation is

$$
\mathscr{G}^{\operatorname{sep}}(\tau ; t) \simeq \mathscr{G}_{s}^{\operatorname{sep}}(\tau ; t)=g^{2}(t) \hat{G}(\tau) .
$$

The following properties of $\hat{G}_{(\tau)}$ are easily proved. Separating real and imaginary parts,

$$
\begin{aligned}
& \hat{\mathscr{G}}(\tau)=\hat{\mathscr{G}}_{R}(\tau)+i \hat{\mathscr{G}}_{I}(\tau), \\
& \hat{\mathscr{G}}_{R}(\tau)=-\hbar^{-1} \int_{0}^{\infty} d \epsilon \rho_{\epsilon}\left|\hat{V}_{-1, \epsilon}\right|^{2} \sin (\epsilon \tau / \hbar), \\
& \hat{\mathscr{G}}_{I}(\tau)=-\hbar^{-1} \int_{0}^{\infty} d \epsilon \rho_{\epsilon}\left|\hat{V}_{-1, \epsilon}\right|^{2} \cos (\epsilon \tau / \hbar)
\end{aligned}
$$

$\hat{\mathscr{G}}_{R}(\tau)$ is an odd function of $\tau$, so $\hat{\mathscr{G}}_{R}(0)=0$. Also

$$
\left.\frac{d \hat{\mathscr{G}}_{R}}{d \tau}\right|_{\tau=0}=-\hbar^{-2} \int_{0}^{\infty} d \epsilon \epsilon \rho_{\epsilon}\left|\hat{V}_{-1, \epsilon}\right|^{2}<0
$$

if the integral converges. $\hat{G}_{I}(\tau)$ is an even function of $\tau$ and $\hat{\mathscr{G}}_{I}(0)<0,\left|d \hat{\mathscr{G}}_{I} / d \tau\right|_{\tau=0}=0$. For all $\tau$,

$$
|\hat{\mathscr{G}}(\tau)| \leq|\hat{\mathscr{G}}(0)|
$$

and

$$
\lim _{\tau \rightarrow \infty} \hat{\mathscr{G}}(\tau)=0 \text {. }
$$

By the same argument as before, the period of time over which $\hat{G}(\tau)$ is significant may typically be only a few atomic units.

The Fourier transform of $\hat{G}(\tau)$ is 


$$
\widehat{G}(\epsilon) \equiv \int_{0}^{\infty} d \tau \exp (i \epsilon \tau / \hbar) \mathscr{G}(\tau)
$$

Again, if $\epsilon$ is given a small positive imaginary part, then we may use (3.22b) in (3.28a) and reverse the order of integration to obtain

$$
\widehat{G}(\epsilon)=\int_{0}^{\infty} d \epsilon^{\prime} \rho_{\epsilon^{\prime}}\left|\hat{V}_{-1, \epsilon^{\prime}}\right|^{2} /\left(\epsilon-\epsilon^{\prime}\right), \operatorname{Im} \epsilon>0 \text {. }
$$

\section{E. A model for $\hat{G}(\tau)$ and $\hat{G}(\epsilon)$}

In the preceding section we obtained $\hat{G}(\epsilon)$ (3.28a), the Fourier transform of $\hat{G}(\tau)$. Equation (3.28b) shows that for $\operatorname{Im} \epsilon>0$, this $\hat{G}(\epsilon)$ is the same quantity that Taylor and Delos obtained in Eq. (2.20) of Ref. 1(a), denoted there as $G(\epsilon)$. Therefore, we can take over many of their results. For $\epsilon<0, \hat{G}(\epsilon)$ is real, negative, and it goes to zero when $\epsilon \rightarrow-\infty$ as $|\epsilon|^{-1}$. For $\operatorname{Re}(\epsilon)>0, \hat{G}(\epsilon)$ is complex, and its imaginary part is given by (3.20), with $\hat{V}_{-1 \epsilon}$ replacing $V_{-1, \epsilon}(t)$. At $\epsilon=0$, the derivative of $\hat{G}(\epsilon)$ is discontinuous.

For more detailed information, we need a model for $\hat{V}_{-1, \epsilon}$. For now we shall use the model given in Ref. 1(a), in which the interaction between the active electron and the neutral $A B$ molecule is replaced by a square well. (We emphasize that this model is quite oversimplified, and it does not constitute an essential part of the present theory.) The resulting $\hat{V}_{\epsilon,-1}$ is given by Eq. (3.7) of Ref. 1(a) (denoted there $V_{E 0}$ ),

$$
\begin{aligned}
V_{\epsilon,-1}= & \frac{2 V_{1}}{(L M N)^{1 / 2}} \\
& \times \frac{k_{1} \sin \left(k_{01} r_{0}\right) \cos \left(k_{1} r_{0}\right)-k_{01} \cos \left(k_{01} r_{0}\right) \sin \left(k_{1} r_{0}\right)}{k_{01}^{2}-k_{1}^{2}},
\end{aligned}
$$$$
k_{1}=\left[\left(2 m / \hbar^{2}\right)\left(\epsilon+V_{0}\right)\right]^{1 / 2} \text {, }
$$$$
k_{01}=\left[\left(2 m / \hbar^{2}\right)\left(E_{0}^{\prime}+V_{0}\right)\right]^{1 / 2} \text {, }
$$$$
M=\left(k_{1}^{2} / k_{2}^{2}\right) \cos ^{2}\left(k_{1} r_{0}\right)+\sin ^{2}\left(k_{1} r_{0}\right),
$$$$
N=r_{0}+\left[V_{0} /\left(E_{0}^{\prime}+V_{0}\right)\right] \sin ^{2}\left(k_{01} r_{0}\right) / k_{02} \text {, }
$$$$
k_{2}=\left[\left(2 m / \hbar^{2}\right) \epsilon\right]^{1 / 2}, \quad k_{02}=\left[-\left(2 m / \hbar^{2}\right) E_{0}^{\prime}\right]^{1 / 2} \text {, }
$$$$
\rho(\epsilon)=\frac{L}{2 \pi}\left(\frac{2 m}{\hbar^{2}}\right)^{1 / 2} \epsilon^{-1 / 2} \text {. }
$$

$V_{0}$ and $r_{0}$ are the depth and width of the potential well, $E_{0}^{\prime}$ is the energy of the bound state in the well, and $V_{1}$ is the strength of the perturbing potential that couples bound to free states. For a numerical calculation, we took

$$
\begin{aligned}
& V_{0}=0.4671, \\
& r_{0}=2.0, \\
& V_{1}=0.09, \\
& E_{0}^{\prime}=-0.0368,
\end{aligned}
$$

where $V_{0}, V_{1}$, and $E_{0}^{\prime}$ are in units of hartree, and $r_{0}$ is in units of bohr.

The resulting $\hat{\mathscr{G}}(\tau)$, calculated by numerical evaluation of the integral (3.22b) is shown in Fig. 1. The integral converges rather slowly, but it is not hard to calculate. The heavy line in Fig. 1 was obtained using an upper limit of $\epsilon=2.5$ hartree. The most important result here is that $\hat{G}(\tau)$ seems to be a rather plain, structureless function having the geneal properties given in the preceding section. If this turns out to hold in general, then reasonable results can be obtained using quite simple models for $\hat{\mathscr{G}}(\tau)$.

When the upper limit of the numerical integration is raised further, to 40 hartree, some structure starts to appear in $\hat{G}(\tau)$ near $\tau=0$; this is shown as the fine line in Fig. 1. We do not believe that this structure is very important; it arises from the very slow oscillations in $\hat{V}_{-1, \epsilon}$ as a function of $\epsilon$, it appears only when we include states that are very high in the continuum $(\epsilon \geq 1 \mathrm{keV}$-detached electrons tend to have energies around $1 \mathrm{eV}$ ), and it may be an artifact of the square-well model. In our calculations, discussed in the following paper, we have taken $\hat{G}(\tau)$ as given by the smooth, heavy line in Fig. 1. The Fourier transform of this $\hat{\mathscr{G}}(\tau)$ is given in Figs. 3 and 4 .

\section{SUMMARY}

Under assumptions given in Sec. II A, processes in which transitions occur from a discrete state to a continuum are described by solving an integro-differential equation (2.23) for $C_{-1}(t)$ (the probability amplitude for finding the system in the discrete state), and using this solution in Eq. (2.13) to find $C_{\epsilon}(t)$ (the amplitude for finding the system in the continuum state of energy $\epsilon)$. To solve (2.23) we need to know the energy gap $\Delta(t)$ between the discrete state and the continuum, and we need to evaluate a propagator $\mathscr{G}\left(t, t^{\prime}\right)$. Energy gaps for several systems have been calculated, ${ }^{16}$ but little is known about the propagator.

We have derived some of the general properties of this propagator, and we have examined some simplifying approximations for it. The two approximations we considered are (i) a short-memory approximation, and (ii) a separable approximation. The short-memory approximation makes use of the fact that the nuclei move slowly, and therefore the time interval $\Delta t\left(\sim a_{0} / v\right)$ over which $V_{\epsilon,-1}(t)$ changes significantly is long compared to the time interval $\Delta \tau \simeq \hbar / \Delta \epsilon$ over which $\mathscr{G}\left(t, t^{\prime}\right)$ is significant. The separable approximation assumes that the important matrix elements $V_{\epsilon,-1}(t)$ have approximately the same time dependence. These two approximations are independent; in any given system, one or both or neither might be applicable.

Using the separable approximation, the propagator can be written approximately in the form (3.1), with $\mathscr{G}(\tau)$ and $g(t)$ behaving as indicated in Figs. 1 and 2. The Fourier transform of $\hat{G}(\tau)$, which turns out to be related to the local-complex-potential, is shown in Figs. 3 and 4 . In the following paper we will develop methods for solving the fundamental integro-differential equation (2.23), and we will show some typical solutions. 


\section{ACKNOWLEDGMENTS}

Portions of this paper are based on the $\mathrm{Ph} . \mathrm{D}$. dissertation by one of us (T.S.W.), College of William and Mary, 1983. Support for this project was provided by the $\mathrm{Na}$ tional Science Foundation and the Jeffress Foundation.

\section{APPENDIX A: DIABATIC AND ADIABATIC REPRESENTATIONS}

\section{Fundamental theorem}

Given a set of coupled equations (2.2) associated with an expansion of the type (2.1), we shall show that representations exist in which $\underline{P}^{R}$ is block-diagonal

$$
\underline{P}^{R}=\left(\begin{array}{cc}
\underline{P}_{\mathrm{I}, \mathrm{I}}^{R} & \underline{0} \\
\underline{0} & \underline{P} \\
\mathrm{II}, \mathrm{II}
\end{array}\right),
$$

so that $P$-matrix elements between selected pairs of states vanish. This is a (rather trivial) generalization of a theorem first established by F. T. Smith.

For $R \rightarrow \infty$, let us divide the basis set $\left\{\phi_{n}(\vec{r} ; R)\right\}$ into two completely arbitrary classes of states, designated as type I and type II, and let us renumber the states such that all states of type I precede all states of type II. Normal$1 y^{17}$

$$
\begin{aligned}
& \lim _{R \rightarrow \infty} \mathscr{V}_{m n}(R)=0 \text { all } m \neq n \\
& \lim _{R \rightarrow \infty} P_{m n}^{R}(R)=0 \text { all } m, n \\
& P_{m m}^{R}(R)=0 \text { all } m, \text { all } R .
\end{aligned}
$$

We consider unitary transformations of the type

$$
\phi_{n}^{\prime}(\overrightarrow{\mathrm{r}} ; \overrightarrow{\mathrm{R}})=\sum_{m} U_{m n}(R) \phi_{m}(\overrightarrow{\mathrm{r}} ; \overrightarrow{\mathbf{R}})
$$

with the restriction

$$
\lim _{R \rightarrow \infty} U_{m n}(R)=\delta_{m n} .
$$

For any $R$, a state $\phi_{n}^{\prime}(\overrightarrow{\mathrm{r}} ; \overrightarrow{\mathbf{R}})$ is said to be of type I (or II) if it correlates asymptotically to a state of type I (or II), i.e., if $\phi^{\prime}(\vec{r} ;|\vec{R}| \rightarrow \infty)$ is of type I (or II).

We may label the states in class I by the dummy index $m_{\mathrm{I}}$ (or $n_{\mathrm{I}}$ ) and those in class II by the dummy index $m_{\mathrm{II}}$ (or $n_{\text {II }}$ ). Then (theorem) there exist $R$-dependent unitary transformations such that for all $m_{\mathrm{I}} n_{\mathrm{I}} n_{\mathrm{II}}$ with $m_{\mathrm{I}} \neq n_{\mathrm{I}}$

$$
\begin{aligned}
& P_{m_{\mathrm{I}} n_{\mathrm{II}}}^{R}=0, \\
& \mathscr{V}_{m_{\mathrm{I}} n_{\mathrm{I}}}=0 .
\end{aligned}
$$

The existence of such representations follows from the well-known transformation rules for $\underline{\mathscr{C}}$ and $\underline{P}^{R}$ :

$$
\begin{aligned}
& \underline{\mathscr{V}}^{\prime}=\underline{U}^{\dagger} \underline{\mathscr{V}} \underline{U}, \\
& \underline{P}^{R^{\prime}}=\underline{U}^{\dagger} \underline{P}^{R} \underline{U}-i \hbar \underline{U} \underline{\dagger}^{\dagger} \frac{d \underline{U}}{d R} .
\end{aligned}
$$

We may construct a representation satisfying (A5) in two steps. First, in the full space of states of types I and II, we can construct $\underline{U}_{1}$ such that $\underline{P}^{R^{\prime}}$ vanishes identically.
The matrix generating this transformation satisfies the equation

$$
i \hbar \frac{d \underline{U}_{1}}{d R}=\underline{P}^{R} \underline{U}_{1}
$$

and the boundary condition (A4) implies a unique solution. In this new representation, matrix elements of $\mathscr{V}$ are generally nonzero. Second, restricting ourselves to the subspace of states of type I, we construct a unitary matrix that diagonalizes $\mathscr{V}_{I, I}$ (the projection of $\mathscr{V}$ onto the "I" subspace). This transformation leads to nonzero $P$ matrix elements within the I subspace, but those connecting I to II still vanish. The result is a representation satisfying (A5).

\section{Interpretation}

In the present case we have one discrete state (type I) and a set of continuum states (type II), so the above theorem establishes that there is a representation in which $P_{-1 \epsilon}^{R}(R)$ vanishes, and couplings between the discrete state and the continuum are represented by $\mathscr{V}_{-1, \epsilon}(R)$.

The theory given in this paper does not, of course, require that $P_{-1, \epsilon}^{R}(R)$ actually vanish, only that it be negligible,

$$
v^{R} P_{-1, \epsilon}^{R}(R) \ll \mathscr{V}_{-1, \epsilon}(R) .
$$

To obtain a basis set satisfying this condition, it is not necessary to use the procedure discussed above. There are good reasons to believe that negative-ion states calculated by the methods of Olson and Liu have this property (A8). Those states were calculated by a kind of "restricted" configuration-interaction method: The zero-order basis functions did not have any dramatic $R$ dependence, and the (square-integrable) basis set excluded very diffuse states. In other words, starting with an approximate partitioning between bound and free states, and using a basis in which the entire $P$ matrix is small, Olson and Liu diagonalized $h_{\mathrm{I}, \mathrm{I}}$, the projection of $h$ onto the bound states. The resulting discrete energy levels often pass right through the continuum, but show avoided crossings with other discrete states. Hence it is reasonable to think that $P_{-1, \epsilon}^{R}(R)$ will be small in this representation.

\section{APPENDIX B: CONTINUITY PROOF}

In this appendix we sketch proofs which give sufficient (but not necessary) conditions that $C_{-1}(t)$ and its first two derivatives, $\mathscr{G}\left(t, t^{\prime}\right)$ and its derivative, and $d \mathscr{I} / d t$ are all continuous.

As stated earlier, it is assumed that $\Delta(t), \rho_{\epsilon}, V_{\epsilon \epsilon}(t)$, and $V_{\epsilon,-1}(t)$ are differentiable functions of $t$. If in addition, for all $t, t^{\prime}$

$$
\left|\rho_{\epsilon} V_{-1, \epsilon}(t) V_{\epsilon,-1}\left(t^{\prime}\right)\right| \leq M_{1}(\epsilon)
$$

and

$$
\int_{0}^{\infty} M_{1}(\epsilon) d \epsilon \text { converges , }
$$

then $\mathscr{G}\left(t, t^{\prime}\right)$ is a continuous function of $t$ (Weierstrass $M$ test $\left.^{18}\right)$, and so is $\mathscr{G}\left(t^{\prime}, t\right)$. If 


$$
\left|\rho_{\epsilon} V_{-1, \epsilon}(t)\right| \leq M_{2}(\epsilon)
$$

and

$$
\int_{0}^{\infty} M_{2}(\epsilon) d \epsilon \text { converges , }
$$

then $\mathscr{I}(t)$ is a continuous function of $t$. It then follows that Eq. (2.16) has solutions $C_{-1}(t)$ which are continuous and differentiable; furthermore, $d C_{-1}(t) / d t$ is also continuous. If

$$
\left|\frac{\partial}{\partial t}\left\{\rho_{\epsilon} V_{-1, \epsilon}(t) \exp \left[-i \Phi_{\epsilon}\left(t, t^{\prime}\right) / \hbar\right] V_{\epsilon,-1}\left(t^{\prime}\right)\right\}\right| \leq M_{3}(\epsilon)
$$

and

$$
\int_{0}^{\infty} M_{3}(\epsilon) d \epsilon \text { converges , }
$$

then $\partial \mathscr{G}\left(t, t^{\prime}\right) / \partial t$ exists and it is continuous. Likewise if

$$
\left|\frac{\partial}{\partial t}\left\{\rho_{\epsilon} V_{-1, \epsilon}(t) \exp \left[-i \Phi_{\epsilon}\left(t, t^{\prime}\right) / \hbar\right]\right\}\right| \leq M_{4}(\epsilon)
$$

and

$$
\int_{0}^{\infty} M_{4}(\epsilon) d \epsilon \text { converges , }
$$

then $d \mathscr{I} / d t$ exists and is continuous.

Differentiating Eq. (2.16) we have

$$
\begin{aligned}
i \hbar \frac{d^{2} C_{-1}(t)}{d t^{2}}= & \frac{d}{d t}\left[\Delta(t) C_{-1}(t)\right]+\mathscr{G}(t, t) C_{-1}(t) \\
& +\int_{t_{0}}^{t} d t^{\prime}\left(\frac{\partial \mathscr{G}\left(t, t^{\prime}\right)}{\partial t}\right) C_{-1}\left(t^{\prime}\right)+\frac{d \mathscr{I}}{d t},
\end{aligned}
$$

and since the right-hand side is continuous, so is the lefthand side.

Derivatives of $\mathscr{G}$ and $\mathscr{I}$ are given by

$$
\begin{aligned}
\frac{\partial \mathscr{G}}{\partial t}=(i \hbar)^{-1} \int_{0}^{\infty} d \epsilon \frac{\partial}{\partial t} & \left\{\rho_{\epsilon} V_{-1, \epsilon}(t)\right. \\
& \left.\times \exp \left[-i \Phi_{\epsilon}\left(t, t^{\prime}\right) / \hbar\right]\right\} V_{\epsilon,-1}(t),
\end{aligned}
$$

$$
\frac{d \mathscr{I}}{d t}=\int_{0}^{\infty} d \epsilon \frac{\partial}{\partial t}\left\{\rho_{\epsilon} V_{-1, \epsilon}(t) \exp \left[-i \Phi_{\epsilon}\left(t, t^{\prime}\right) / \hbar\right]\right\} C_{\epsilon}\left(t_{0}\right),
$$

provided that the integrals converge.

\section{APPENDIX C: CONVERGENCE PROOF}

We give here a proof that the quantity $\widehat{C}_{-1}(t)$, defined in Eq. (2.25), approaches a finite limit as $t \rightarrow \infty$. A sufficient (but by no means necessary) condition for this result is that

$$
\lim _{T \rightarrow \infty} \int_{t_{0}}^{T} d t \int_{t_{0}}^{t} d t^{\prime}\left|\mathscr{G}\left(t, t^{\prime}\right)\right|<\infty .
$$

Under this condition, the proof is simple. The existence of the limit (C1) implies that for any $\epsilon$ there exists a $T^{*}$ such that

$$
\int_{T_{1}}^{T_{2}} d t \int_{t_{0}}^{t} d t^{\prime}\left|\mathscr{G}\left(t, t^{\prime}\right)\right|<\epsilon
$$

for all $T_{1}, T_{2}$ greater than $T^{*}$. Without loss of generality, let us take $T_{2}>T_{1}$. Now

$$
\begin{aligned}
& \left|\widehat{C}_{-1}\left(T_{2}\right)-\widehat{C}_{-1}\left(T_{1}\right)\right|=\left|\int_{T_{1}}^{T_{2}}\left[\frac{d \hat{C}_{-1}}{d t}\right) d t\right| \\
& \leq \int_{T_{1}}^{T_{2}}\left|\frac{d \hat{C}_{-1}}{d t}\right| d t \\
& \frac{d \widehat{C}_{-1}(t)}{d t}=\exp \left[i \int_{t_{0}}^{t} \Delta\left(t^{\prime \prime}\right) d t^{\prime \prime} / \hbar\right] \\
& \times \int_{t_{0}}^{t} \mathscr{G}\left(t, t^{\prime}\right) C_{-1}\left(t^{\prime}\right) d t^{\prime}, \\
& \left|\frac{d \widehat{C}_{-1}(t)}{d t}\right| \leq \int_{t_{0}}^{T}\left|\mathscr{G}\left(t, t^{\prime}\right)\right| d t^{\prime},
\end{aligned}
$$

so

$$
\left|\hat{C}_{-1}\left(T_{2}\right)-\hat{C}_{-1}\left(T_{1}\right)\right| \leq \int_{T_{1}}^{T_{2}} d t \int_{t_{0}}^{t} d t^{\prime}\left|\mathscr{G}\left(t, t^{\prime}\right)\right|<\epsilon
$$

for all $T_{1}, T_{2}$ greater than $T^{*}$. By the Cauchy criterion, ${ }^{18}$ $\widehat{C}_{-1}(t)$ approaches a finite limit as $t \rightarrow \infty$.
${ }^{1}$ (a) R. D. Taylor and J. B. Delos, Proc. R. Soc. London, Ser. A 379, 179 (1982); (b) 379, 209 (1982); (c) T. S. Wang and J. B. Delos, J. Chem. Phys. 79, 4306 (1983); (d) following paper, Phys. Rev. A 29, 552 (1984). To understand the present paper, it is not necessary to read the earlier ones in detail. For some readers, however, it might be helpful to look at the first half of Ref. 1(a), pp. 179-192; (e) T. S. Wang, Ph.D. thesis, College of William and Mary, 1983.

${ }^{2}$ S. K. Lam, J. B. Delos, R. L. Champion, and L. D. Doverspike, Phys. Rev. A 9, 1828 (1974); R. L. Champion and L. D. Doverspike, J. Chem. Phys. 65, 2482 (1976); Phys. Rev. A 13, 609 (1976); R. L. Champion, L. D. Doverspike, and S. K. Lam, ibid. 13, 617 (1976); B. T. Smith, W. R. Edwards, III,
L. D. Doverspike, and R. L. Champion, ibid. 18, 945 (1978); S. E. Haywood, L. D. Doverspike, R. L. Champion, E. Herbst, B. K. Annis, and S. Datz, J. Chem. Phys. 74, 2845 (1981); L. D. Doverspike, B. T. Smith, and R. L. Champion, Phys. Rev. A 22, 393 (1980); M. S. Huq, L. D. Doverspike, R. L. Champion, and V. A. Esaulov, J. Phys. B 15, 951 (1982); M. S. Huq, D. S. Fraedrich, L. D. Doverspike, R. L. Champion, and V. A. Esaulov, J. Chem. Phys. 76, 4952 (1982); B. K. Annis, S. Datz, R. L. Champion, and L. D. Doverspike, Phys. Rev. Lett. 45, 1554 (1980).

${ }^{3}$ C. de Vreugd, R. W. Wijnaendts von Resandt, J. B. Delos, and

J. Los, Chem. Phys. 68, 261 (1982); 68, 275 (1982).

4J. S. Risley, Phys. Rev. A 10, 731 (1974); J. S. Risley and R. 
Geballe, ibid. 9, 2485 (1974).

${ }^{5}$ V. Esaulov, D. Dhuicq, and M. Barat, J. Phys. B 11,1267 (1978); J. Fayeton, D. Dhuicq, and M. Barat, ibid. 11,1049 (1978); J. L. Montmagnon, V. Esaulov, J. P. Grouard, R. I. Hall, M. Landau, F. Pichou, and C. Scherman, ibid. 16, L143 (1983); Vu Ngoc Tuan, J. P. Gauyacq, and V. Esaulov, ibid. 16, L95 (1983).

${ }^{6}$ U. Fano, Phys. Rev. 124, 1866 (1961).

${ }^{7}$ Yu. N. Demkov and V. I. Osherov, Zh. Eksp. Teor. Fiz. 프, 1589 (1967) [Sov. Phys._JETP 26, 916 (1968)]; Yu. N. Demkov, Dok. Akad. Nauk SSSR 166, 1076 (1966) [Sov. Phys.Dok1. 11, 138 (1966)].

${ }^{8}$ (a) For a review, see J. B. Delos, Rev. Mod. Phys. 프, 287 (1981); J. B. Delos and W. R. Thorson, J. Chem. Phys. 70, 1774 (1979). (b) Translation factors are also discussed by $\mathrm{D}$. R. Bates and R. McCarroll, Proc. R. Soc. London Ser. A 245, 175 (1958); S. B. Schneiderman, and A. Russek, Phys. Rev. $\underline{181}, 311$ (1969); T. A. Green, Phys. Rev. A $\underline{23}, 519$ (1981); $\underline{23}$, 532 (1981).

${ }^{9}$ In Ref. $8(\mathrm{a})$, it was shown that the $\underline{\overrightarrow{\mathrm{A}}}$-matrix, which contains the modifications to $\underline{\overrightarrow{\mathbf{P}}}$ that arise from ETF's, tends to be small for degeneracy-mediated transitions, provided that the degeneracy occurs at small to moderate internuclear distances.

${ }^{10}$ D. M. Chase, Phys. Rev. 104, 838 (1956); D. E. Golden, N. F. Lane, A. Temkin, and E. Gerjuoy, Rev. Mod. Phys. $\underline{43}, 642$ (1971).

${ }^{11}$ A. U. Hazi, T. N. Rescigno and M. Kurilla, Phys. Rev. A $\underline{23}$, 1089 (1981); M. Berman, H. Estrada, L. S. Cederbaum, and W. Domcke, ibid. $\underline{28}, 1363$ (1983).

12It has been claimed [H. S. Taylor (private communication)] that free-free transitions were incorporated into the theory developed by Hazi et al. (Ref. 11). This is incorrect. It was stated very clearly in that paper that the adiabatic-nuclei approximation was used for the nonresonant part of the scattering.

${ }^{13}$ We should mention that even if free-free transitions cannot be neglected, much of the present theory still holds, with appropriate modifications. For example, an equation like (2.16) can still be derived, but $\mathscr{G}$ and $\mathscr{I}$ are more complicated functions.

${ }^{14}$ F. Fiquet-Fayard, Vacuum 24, 533 (1974).

${ }^{15}$ It might be mentioned that although projection operators do not appear in this paper, the theory given here can be written as a time-dependent analog of the Feshbach projectionoperator method. This time-dependent analog has not seen much use in quantum mechanics, but it has been extensively used in statistical mechanics. See, for example, R. Zwanzig, J. Chem. Phys. 33, 1338 (1960); also Lectures in Theoretical Physics, edited by W. E. Brittin, B. W. Downs, and J. Downs (Interscience, New York, 1961), Vol. III, pp. 106-141.

${ }^{16}$ R. E. Olson and B. Liu, Phys. Rev. A 17, 1568 (1978); 20, 1344 (1979); 22, 1389 (1980); J. Chem. Phys. 73, 2817 (1980); J. P. Gauyacq, J. Phys. B 13, L501 (1979); 12, L387 (1979); 13, 4417 (1980).

${ }^{17}$ If electron-translation factors are not included, some $P$-matrix elements may have infinite range. We already stated that in the cases of interest to us, this can probably be repaired by a simple change of the origin of coordinates. More generally (Ref. 8), one must take additional corrections into account, and a diabatic representation is one in which $P+A$ is negligible.

${ }^{18}$ D. Widder, Advanced Calculus, 2nd ed. (Prentice-Hall, Englewood Cliffs, 1961); W. Kaplan, Advanced Calculus (AddisonWesley, Reading, Mass., 1952). 